REUSE-BASED METHODOLOGIES AND TOOLS IN THE DESIGN OF ANALOG AND MIXED-SIGNAL INTEGRATED CIRCUITS 


\section{Reuse-Based Methodologies and Tools in the Design of Analog and Mixed-Signal Integrated Circuits}

by

R. CASTRO-LÓPEZ

IMSE-CNM-CSIC,

Spain

F.V. FERNÁNDEZ

University of Seville, IMSE-CNM-CSIC,

Spain

O. GUERRA-VINUESA

University of Seville,

Spain

and

Á. RODRÍGUEZ-VÁZQUEZ University of Seville,

Spain

望 Springer 
A C.I.P. Catalogue record for this book is available from the Library of Congress.

ISBN-10 1-4020-5126-3 (HB)

ISBN-13 978-1-4020-5126-5 (HB)

ISBN-10 1-4020-5139-5 (e-book)

ISBN-13 978-1-4020-5139-5 (e-book)

Published by Springer,

P.O. Box 17, 3300 AA Dordrecht, The Netherlands.

www.springer.com

Printed on acid-free paper

All Rights Reserved

(C) 2006 Springer

No part of this work may be reproduced, stored in a retrieval system, or transmitted

in any form or by any means, electronic, mechanical, photocopying, microfilming, recording

or otherwise, without written permission from the Publisher, with the exception

of any material supplied specifically for the purpose of being entered

and executed on a computer system, for exclusive use by the purchaser of the work.

Printed in the Netherlands. 
Rafael Castro López

To my family and friends

Francisco V. Fernández

To Eli, Judit, and Nuria

Óscar Guerra Vinuesa

To my wife and daughters Ángel Rodríguez Vázquez

To my former doctoral students and current friends 


\section{Contents}

Preface $\quad$ xi

1 INTRODUCTION 1

1 Problem overview: The design gap 1

1.1 Evolution of the semiconductor industry 1

1.2 The design gap 5

1.2.1 Time-to-market 7

1.2.2 Design complexity 8

$\begin{array}{ll}1.3 \text { Analog design automation } & 10\end{array}$

2 Problem definition $\quad 14$

2.1 Hierarchy, abstraction, and views 14

2.2 The AMS design flow 16

3 Summary 25

2 A REUSE-BASED DESIGN FRAMEWORK FOR ANALOG ICs 27

1 Design automation $\quad 27$

1.1 Preliminary definitions 28

1.2 The two sides of automation 29

1.2.1 Knowledge-based synthesis 30

1.2.2 Optimization-based synthesis 31

1.2.3 Quality metrics for analog synthesis 34

1.3 Knowledge versus optimization-based synthesis 35

2 Circuit reuse 38

2.1 Preliminary definitions $\quad 38$

2.2 Digital design reuse 41

2.3 Analog design reuse $\quad 42$

2.4 Other approaches to analog reuse 45 
3 The reuse-based design framework

3.1 The analog reusable block $\quad 51$

3.2 The design reuse flow 54

3.2.1 Adopted synthesis approaches 54

3.2.2 The top-down path 56

3.2.3 The bottom-up path 58

3.2.4 The role of the analog reusable block $\quad 59$

3.3 The design for reusability methodology 60

4 Summary 62

3 THE ANALOG REUSABLE BLOCK: BEHAVIORAL FACET 63

1 Introduction: Why behavioral descriptions? 63

1.1 Analog behavioral modeling taxonomy 66

2 Facing design reuse $\quad 68$

2.1 The design reuse flow: top-down electrical synthesis $\quad 68$

2.2 The design reuse flow: bottom-up verification 72

2.3 Characteristics of the behavioral facet of the AMS

3 Case study: a quadrature DA transmit interface $\quad 76$

3.1 System description $\quad 76$

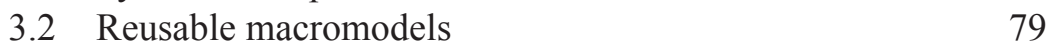

4 Summary $\quad 88$

4 THE ANALOG REUSABLE BLOCK: STRUCTURAL FACET 89

1 Introduction 89

1.1 Adopted sizing approach $\quad 92$

2 Design knowledge encapsulation 93

2.1 Netlist-related elements $\quad 96$

$\begin{array}{ll}2.1 .1 & \text { Design variables }\end{array}$

2.1.2 Constraints 100

2.2 Testbench setups 105

2.2.1 Performance feature elements 106

2.2.2 Peripheral setup elements 109

2.2.3 Component model and process data elements 111

2.2.4 Design variables, dependent variables, and constraints

3 Practical aspects of structural view reuse 117

4 Summary 122 
1 Introduction

2 Layout retargeting 125

2.1 Device mismatch 125

2.2 Loading effects 128

2.3 Coupling effects 129

2.4 Reliability 130

2.5 Area occupation 131

3 Layout migration 132

4 Analog layout strategies $\quad 135$

4.1 Optimization-driven approaches 138

4.2 Knowledge-driven approaches 140

5 Automated layout generation for design reuse 142

6 Layout template: definition and properties 144

7 Creating the layout template 150

7.1 Device-level layout generation: primitives 158

7.1.1 Reuse: migration issues 159

7.1.2 Reuse: retargeting issues 160

7.1.3 PDLP coding 162

7.2 Device-level layout generation: blocks 170

7.2.1 Reuse: migration issues 172

$\begin{array}{ll}7.2 .2 & \text { Reuse: retargeting issues } \\ 7.2 .3 & 172\end{array}$

7.2.3 PDLB coding 173

$\begin{array}{lll}7.3 & \text { Layout template generation } & 182\end{array}$

7.3.1 Reuse: migration issues 183

7.3.2 Reuse: retargeting issues 185

$\begin{array}{ll}\text { 7.3.3 Layout template coding } & 187\end{array}$

8 Practical implementation of layout-reusable analog blocks 194

8.1 Layout languages 194

8.2 Implementation examples 196

9 Summary 205

6 DESIGN EXAMPLES AND SILICON PROTOTYPE 207

1 Introduction 207

2 The demonstration vehicle 209

2.1 Application area and rationale for architecture selection 210

2.2 System specifications and specifications of the analog back-end

2.3 Hierarchy of the analog back-end 214 
2.4 Analysis of the analog back-end

3 Reusable blocks

3.1 Reusable blocks: opamps

3.2 Reusable blocks: analog back-end

4 Design examples

4.1 Design example (I): design retargeting and migration of the opamp

4.1.2 Opamp migration to process B $(0.5 \mu \mathrm{m})$

4.2 Design example (II): GSM retargeting of the analog back-end

4.3 Design example (III): multi-standard retargeting of the analog back-end

4.4 Automation prototype

5 Silicon prototype

6 Costs and benefits 287

7 Summary

7 LAYOUT-AWARE CIRCUIT SIZING

1 Introduction 289

2 Geometrically constrained sizing 290

2.1 Formulation of the problem 292

2.2 Review of previous approaches 294

2.3 An integrated approach $\quad 300$

2.4 Experimental results $\quad 312$

3 Parasitic-aware sizing $\quad 324$

$\begin{array}{lll}3.1 & \text { Layout parasitics } & 324\end{array}$

3.2 Extraction methods 329

3.3 Extraction of parasitics in the design process 332

3.4 Demonstration of the parasitic-aware design flow $\quad 337$

4 Summary 345

APPENDIX A: Analog and Mixed-Signal Layout Rules 347 


\section{Preface}

Whether the widely cited Moore's Law -forecasting that the number of transistors that can be fit into a chip roughly doubles every two years- has actually represented a roadmap the semiconductor industry has struggled to comply with or a long-term prediction proven true, the fact is that this industry has accomplished spectacular breakthroughs in past decades, pervasively impacting most aspects of everyday life.

Despite these breakthroughs, the spiraling cost of integrated circuit (IC) design is slowly but surely wrapping a noose around the neck of the semiconductor industry. The economics of building today's even-more-complex ICs under even-more-stringent time-to-market requirements (perhaps the most impelling forces in modern semiconductor industry) are already so daunting that the 2003 ITRS report singled out the cost of chip design as "the greatest threat to the continuation of the semiconductor roadmap". The resulting design productivity gap -the gulf between what is possible to manufacture and what is possible to design- will certainly widen, slowing down this industry's phenomenal growth.

In the past, the industry has extracted itself from design cost traps by finding a way to automate portions of the IC design process, allowing designers to become more productive and driving costs back down. Today, the problem cannot be tackled by still relying on 20-year-old design automation technology or by simply hiring more qualified engineers. The design community believes that powerful computer-aided design (CAD) tools and capable CADbased methodologies do not suffice in order to successfully and utterly bridge the design gap, but that some kind of design paradigm shift must be urgently put on stage.

In this sense, reuse-based design practices are regarded as a promising solution, and concepts such as IP Block, Virtual Component, and Design 
Reuse have become commonplace thanks to the significant advances in the digital arena. Although far from being completely settled, an important market has flourished around digital reuse that furnishes design companies with solutions to noticeably improve their productivity rate.

When it comes to analog and mixed-signal (AMS) design, the scenario is, unfortunately, not that optimistic. The current level of AMS CAD, lagging several generations behind digital design automation partly because of the very nature of AMS design -more subtle, hierarchically loose, and handicraftdemanding-, partly because of the comparatively smaller amount of R\&D dedicated to AMS CAD, and the huge heterogeneity of AMS circuits, has so far hindered a similar level of consensus and development on AMS reusebased design, frequently influencing the idea that inheriting digital reuse concepts is impractical or simply unrealizable. It is necessary to remark, however, the importance of improving AMS design productivity: despite the relatively smaller silicon area dedicated to AMS circuitry, the time needed to design this circuitry dominates, in most cases, the total design time. Therefore, any research ultimately targeted at the improvement of the design productivity of ICs should consider AMS design productivity as a goal priority as well. Otherwise, design productivity will eventually get stuck on the AMS design bottleneck.

In this scenario, the research reported in this book tries to demonstrate not only that reuse-based design in the AMS arena is possible, but also that by following such a design paradigm and making use of appropriate CAD tools, techniques, and methods, it is possible to break through the bottlenecks of AMS design and enhance the design productivity. The concept of reuse here cannot be simply based on plug-in pre-designed, fixed circuit blocks out of a design repository, but rather on recycling these blocks; that is, adopting a flexible methodology by which a circuit can be easily and seamlessly adapted to different design specifications, different environments, and different technology nodes and foundries, thereby completing a AMS design project in time.

This book presents a framework for the reuse-based design of AMS integrated circuits. This framework is founded on three key elements:

- first, a CAD-supported hierarchical design flow that facilitates the incorporation of AMS reusable blocks. Thanks to this design reuse flow, overall design time can be reduced and increasing AMS design complexity can be efficiently managed;

- second, a complete and clear definition of the AMS reusable block. Such definition is structured into three separate facets or views: the 
behavioral, structural, and layout facets. Throughout block reuse, design information flows from one facet to another, progressively adapting it to the targeted performance and technology. Each facet is devised to suit a stage of the design reuse flow, at its corresponding hierarchical level. In this way, the behavioral and structural facets are used for top-down electrical synthesis and bottom-up verification, and the layout facet is used for bottom-up physical synthesis;

- third, the set of methods, tools, and guidelines composing the design for reusability methodology, which allows producing fully reusable AMS blocks. This methodology relies on intensive facet parameterization as well as on the capture and encapsulation of design knowledge within each facet.

Although the book undertakes the problem from a general perspective, covering all different stages of the design flow, it makes special emphasis on AMS physical design reuse, as this is one of the most (if not the most) crucial, knowledge-intensive stages of the AMS design flow, thus posing a greater challenge to reuse-based design.

The framework is completed with a synthesis technique that aims at speeding up the design process of AMS ICs by reducing the time-consuming, errorprone iterations between electrical and physical synthesis, traditionally considered as non-miscible design stages. In this so-called layout-aware electrical synthesis, a simulation-based optimization algorithm explores the design space while specific and detailed information of the circuit layout -its geometric features and its layout-induced degradation on the circuit's performance- is used to improve the synthesized solution, yielding a correctby-construction physical implementation of the circuit during the first pass.

The framework has been put into practice and assessed on a well-known, commercial design environment (Design Framework II from Cadence $\left.{ }^{\circledR}\right)$. Furthermore, the framework has been validated through an industrial-scale, functional silicon prototype, consisting in an universal IQ transmit interface for wireless communications.

The contents of this book are organized in seven chapters as follows.

Chapter 1 introduces the problem rationale by examining the evolution of the semiconductor industry, analyzing the current challenges, and delving into the causes of the design productivity gap. To set the background of the research, the chapter then proceeds to clearly define the problem by resorting to several key concepts such as hierarchy, abstraction level, and circuit view, 
and answering the question of why traditional AMS design methodologies cannot solve it.

Chapter 2 reviews the current state of AMS design automation technology and, in the light of this revision, presents the reuse-based design paradigm. The digital reuse scenario is then examined in order to give insight into the differing requirements of AMS reuse. Afterwards, the chapter surveys the state-of-the-art of AMS reuse-based design. Last, the reuse-based design framework proposed in this book is described.

Chapters 3, 4, and 5 respectively describe the behavioral, structural, and layout facets of the AMS reusable block. The description of each facet follows a three-part structure: what is and what is the facet used for, what requirements does reuse-based design impose on the facet, and how reusability can be built on the facet. Accompanying the descriptions, each chapter contains detailed illustrative examples.

Chapter 6 reports the experimental demonstration of the validity of the reuse-based design framework. This chapter comprises several design experiments, as well as the description and experimental verification results of the silicon prototype mentioned above, whose analog section has been designed under the proposed framework.

Finally, Chapter 7 presents and demonstrates the layout-aware synthesis technique.

The considerations presented in Chapters 4, 5, and 7 are complemented in Appendix A.

The work presented in this book has been partially supported by the TEC2004-01752 Project (funded by the Spanish Ministry of Education and Science with support from the European Regional Development Fund) and the MEDEA+2A101-SPIRIT Project. 